\title{
Naturlatexallergie sicher nachweisen
}

\author{
Auf der Jahrestagung der American Academy of \\ Asthma, Allergy and Immunology (AAAAI) in \\ San Francisco vom 21.-26. Februar 1997 unternahm \\ R. G. Hamilton, Baltimore, eine kurze Bestands- \\ aufnahme zum Stellenwert verschiedener Methoden \\ der In-vitro-Diagnostik bei Naturlatexallergie.
}

eit der Identifikation des Immunglobulins $\mathrm{E}$ ( $\mathrm{IgE}$ ) als primärer Auslöser einer allergischen Soforttypreaktion im Jahre 1966 wurden in den letzten 30 Jahren weitere serologische Laborverfahren entwickelt, die eine Diagnose einer allergischen Erkrankung erleichtern. Von allen Methoden hat sich der Nachweis spezifischer IgE-Antikörper als wichtigster Test etabliert. Er dient zur Bestätigung der Ergebnisse der Hauttestung und bleibt bei fehlenden zugelassenen Naturlatexallergen-Hauttestreagenzien in den USA der einzige sichere und reproduzierbare diagnostische Test. Aufgrund unterschiedlicher Qualität der Naturlatexallergenextrakte sowie methodischer Unterschiede sind die Ergebnisse verschiedener Testverfahren zum Nachweis von latexspezifischem $\operatorname{IgE}$ quantitativ nicht vergleichbar, weisen qualitativ jedoch eine hohe Sensitivität auf.

Demgegenüber kann die Messung des Gesamt-Serum-IgE's aufgrund großer Überlappungen nur als unspezifischer Marker einer atopischen Disposition gewertet werden. Er ist für den Nachweis einer Naturlatexallergie ebenso wenig von Bedeutung wie die Bestimmung von antigenspezifischem $\mathrm{IgG}$, welches lediglich Hinweise auf die Exposition eines Patienten mit einem Allergen geben kann. Tryptase und Eosinophil Cationic Protein (ECP) als Marker einer Mastzell- bzw. Eosinophilenaktivierung werden hauptsächlich für Forschungsfragen bestimmt und können ggf. zum unspezifischen Nachweis einer inflammatorischen oder anaphylaktoiden Reaktion eingesetzt werden. Da auch der Nachweis von allergenen Naturlatexproteinfraktionen durch immunelektrophoretische Verfahren der Forschung vorbehalten ist, bleibt derzeit die Messung spezifischer IgEAntikörper die wesentliche Laboruntersuchung zum Nachweis einer Naturlatexallergie.

Historisch war der Pharmacia RAST der erste Immunassay zum Nachweis von spezifischem IgE. Eine Papierscheibe mit kovalent gebundenem Allergen bindet spezifisches IgE aus dem Patientenserum. In einer zweiten Inkubation wird das spezifische IgE nach dem Waschen durch radiomarkiertes Anti-IgE quantifiziert. Die Basis dieses Prinzips ist auch bei den anderen Testverfahren weitgehend unverändert. Variationen bestehen jedoch in der Antigenpräsentation in einer flüssigen Testphase, dem Einsatz von anderen Detektionsverfahren (FEIA, EIA, Chemilumineszenz) und der Kalibration und Bewertung des Ergebnisses.

\section{Problem: Standardisierung der Allergenxtrakte}

Die wesentliche Komponente hingegen stellt der Allergenextrakt dar, welcher für diesen Test eingesetzt wird. Er sollte alle wichtigen klinisch-relevanten Allergene beinhalten und adäquate Stabilität und Immunreaktivität gewährleisten. Naturlatexextrakte weisen jedoch eine starke Heterogenität durch biologische Variation der Allergenquelle, durch die Schwankungen zwischen verschiedenen Chargen im Extraktionsproze $\beta$, unterschiedliche Bindung zur Trägerphase und variable Stabilität auf. An möglichen Naturlatexallergenquellen kommen nicht-ammoniertes Rohlatex (NAL), ammoniertes Latex (AL) und naturlatexenthaltende Endprodukte (z.B. Handschuhmaterial) zum Einsatz. Diese Quellen enthalten eine unterschiedliche Allergenzusammensetzung hinsichtlich Qualität und Quantität. Als die besten Allergenquellen werden NAL und die Endprodukte angesehen, mit mehr als $90 \%$ iger Konkordanz der Befunde zwischen unterschiedlichen Laboratorien. Eine Voraussetzung für eine verläßliche Interpretation von Ergebnissen ist, daß alle kommerziellen Testverfahren hinsichtlich Allergenmaterial, aber auch bezüglich der Kalibration, Interpolationsmethoden und Angabe der Antikörperklassen vergleichbar sind. Zur Zeit sind in den USA nur die Assays von Pharmacia und DPC zum Nachweis von latexspezifischen $\operatorname{IgE}$ kommerziell zugelassen. In einer Studie, in der 11 verschiedene Methoden in 75 Laboratorien untersucht wurden, wurde in einer gut charakterisierten Studienpopulation (inkl. Provokationstest) die qualitative Sensitivität überprüft. In $97,3 \%$ wurden bei Vorhandensein einer Naturlatexallergie spezifische Antikörper festgestellt (JACI 1996; 98: 872-83). Eine qualitative Vergleichbarkeit der Stärke der Allergie in Stärkeklassen ist jedoch aufgrund der o.a. Unterschiede zwischen den verschiedenen Methoden nicht gegeben.

Zusammenfassend stellt die Bestimmung spezifischer IgE-Antikörper auf Naturlatex die einzige reproduzierbare und sichere Labormethode zum Nachweis einer Naturlatexallergie dar. Die Probleme hinsichtlich der Standardisierung der Allergenextrakte und der Vergleichbarkeit der quantitativen Testergebnisse können jedoch nicht übersehen werden.

\section{Korrespondenzadresse:}

Dr. med. K. Brockow, Klinik und Poliklinik für Dermatologie und Allergologie am Biederstein, TUM, Biedersteiner Str. 29, D-80802 München. 